\title{
SARS-COV-2 VACCINE IN PATIENTS WITH RHEUMATOID ARTHRITIS: ATTENUATED RESPONSE INDUCED BY SPECIFIC DMARD, DMARD COMBINATION AND PREDNISONE
}

Ana Cristina de Medeiros Ribeiro ${ }^{1, \star}$, Karina Bonfiglioli ${ }^{1}$, Diogo Domiciano ${ }^{1}$, Andrea Shimabuco ${ }^{1}$, Henrique Carriço1, Carla Saad ${ }^{1}$, Emily Yuki ${ }^{1}$, Sandra Gofinet Pasoto ${ }^{1}$, Tatiana Pedrosa ${ }^{1}$, Carlo Renner Araújo ${ }^{1}$, Matheus Rodrigues Silva ${ }^{1}$, Tatiane Lie Nakai ${ }^{1}$, Guilherme Balbi ${ }^{1}$, Clóvis Silva ${ }^{1}$, Nádia Aikawa ${ }^{1}$, Eloisa Silva Dutra de Oliveira Bonfá ${ }^{1}$

1.Universidade de São Paulo, São Paulo (SP), Brazil.

*Corresponding author: anademedeiros@yahoo.com

\section{BACKGROUND}

Due to the COVID-19 pandemic, there is a pressing need to evaluate the immune response to SARS-CoV-2 vaccines in rheumatoid arthritis (RA) patients, and identify the factors that can affect the immunogenicity in such population.

\section{METHODS}

This is a phase 4 prospective study that analyzed immunogenicity/safety of two doses of inactivated SARS-CoV-2 vaccine (CoronaVac) in 260 seronegative RA patients in comparison to a group of 104 controls (CG), in a 5:2 ratio. Seroconversion (SC) of anti-SARS-CoV-2 S1/S2 IgG and the positivity of neutralizing antibodies (NAb) were assessed 28 days after the first dose (D28) and 6 weeks after the second dose (D69), while vaccine adverse events (AEs) were recorded at each visit. Disease activity status measured by clinical disease activity index (CDAl) and C reactive protein (CRP)-based disease activity score with 28 joints (DAS28-CRP), and use of disease modifying antirheumatic drugs (DMARD) were assessed at recruitment using the last electronic record. Statistical significance was set at $p<0.05$.

\section{RESULTS}

The groups had comparable median (interquartile range - IQR) ages (59 [50-65] vs. 58 [49.8-64], $p=0.483$ ) and female (both $90.4 \%$; $p$ > 0.999) predominance. Median (IQR) disease duration was 19 (11-26) years. Most of patients had rheumatoid factor positivity (77.8\%) and anti-CCP positivity (69.7\%). The median (IQR) baseline CDAI was 8.5 (4-16) and DAS28-CRP was 2.9 (2.2-4). Rheumatoid arthritis patients had lower SC at D28 (10.8\% vs. 30.1\%; $p<0.001)$ and D69 (61.8\% vs. 94.2\%; $p<0.001)$ in comparison to CG. Similarly, RA patients also had lower positivity for NAb at D28 (13.9\% vs. 32\%; $p<0.001)$ and D69 (45\% vs. $78.6 \% ; p<0.001)$. Univariate analysis pointed older age $(p<0.001)$, methotrexate $(p=0.003)$, abatacept $(p<0.001)$ and the combination of $\geq 2$ DMARDs ( $p=0.019$ ) as associated with absence of SC, while older age $(p<0.001)$, prednisone $(p=0.045)$ and abatacept ( $p=0.038$ ) were associated with absence of NAb. In contrast, sulfasalazine ( $p=0.035)$ was positively associated with SC. Baseline disease activity did not interfere with immunogenicity parameters $(p>0.05)$. After multivariate analyses, factors independently related to SC were: older age (odds ratio, $\mathrm{OR}=0.79$ [0.70-0.89] for each 5-year interval; $p<0.001$ ), methotrexate use $(O R=0.50[0.28-0.91] ; p=0.024)$, abatacept use $(O R=0.36[0.18-0.70] ; p=0.003)$, combination of $\geq 2$ DMARDs $(\mathrm{OR}=0.50$ [0.26-0.97]; $p=0.041]$, and sulfasalazine use (OR 3 [1.04-8.75]; $p=0.043)$. Regarding the presence of NAb, age over 60 years $(O R=0.54[0.33-0.90] ; p<0.018)$ and prednisone use $(O R=0.57[0.33-0.99] ; p<0.046)$ were negatively related to presence of NAb. No moderate/severe adverse event occurred.

\section{CONCLUSION}

We have observed that RA patients have a low/moderate reduced immunogenicity to SARS-CoV-2 vaccine. The novel evidence that specific DMARD, DMARD combination and prednisone are the most relevant factors to impair vaccine antibody response in this disease reinforces the need of a distinct approach to improve vaccine response in this population.

\section{KEYWORDS}

SARS-CoV-2 vaccine, Rheumatoid arthritis, Immunogenicity. 